\title{
Apple intake and cancer risk: a systematic review and meta-analysis of observational studies
}

\author{
Roberto Fabiani ${ }^{1, *}$, Liliana Minelli ${ }^{2}$ and Patrizia Rosignoli ${ }^{1}$ \\ 'Department of Chemistry, Biology and Biotechnology, University of Perugia, Via del Giochetto, 06126 Perugia, \\ Italy: ${ }^{2}$ Department of Experimental Medicine, University of Perugia, Perugia, Italy
}

Submitted 14 September 2015: Final revision received 25 January 2016: Accepted 4 February 2016: First published online 22 March 2016

\begin{abstract}
Objective: Conflicting results on the association between fruit consumption and cancer risk have been reported. Little is known about the cancer preventive effects of different fruit types. The present meta-analysis investigates whether an association exists between apple intake and cancer risk.

Design: Relevant observational studies were identified by literature search (PubMed, Web of Science and Embase). A random-effect model was used to estimate the cancer risk in different anatomical sites. Between-study heterogeneity and publication bias were assessed using adequate statistical tests.

Results: Twenty case-control (three on lung, five on colorectal, five on breast, two on oesophageal, three on oral cavity, two on prostate and one each on pancreas, bladder, larynx, ovary, kidney and brain cancer) and twenty-one cohort (seven on lung, two on colorectal, three on breast and one each on oesophageal, pancreas, bladder, kidney, endometrial, head-neck, urothelial and stomach cancer) studies met the inclusion criteria. Comparing the highest $v$. lowest level of apple consumption, the reduction of lung cancer risk was statistically highly significant in both case-control (OR $=0.75 ; 95 \%$ CI 0.63, 0.88; $\left.P=0.001, I^{2}=0 \%\right)$ and cohort studies (relative risk $=0.89 ; 95 \%$ CI 0.84, 0.94; $P<0.001, I^{2}=53 \%$ ). Instead, in the case of colorectal $\left(\mathrm{OR}=0.66 ; 95 \%\right.$ CI $\left.0.54,0.81 ; P<0.001, I^{2}=55 \%\right)$, breast $\left(\mathrm{OR}=0.79 ; 95 \% \mathrm{CI} 0.73,0.87 ; P<0.001, I^{2}=1 \%\right)$ and overall digestive tract $\left(\mathrm{OR}=0.50 ; 95 \%\right.$ CI 0.36, 0.69; $\left.P<0.001, I^{2}=90 \%\right)$ cancers a significant preventive effect of apples was found only in case-control studies while prospective studies indicated no effect. No evidence of publication bias could be detected for colorectal, oral cavity, oesophageal and breast cancer. However, some confounding effects may be present and related to the consumption of other fruit which have not been considered as adjusting factors.

Conclusions: The present meta-analysis indicates that consumption of apples is associated with a reduced risk of cancer in different anatomical sites.
\end{abstract}

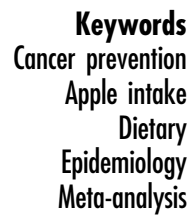

Cancer is a chronic degenerative disease causing major morbidity and mortality in Western countries. It has been estimated that in the year 2012, 14.1 million new cancer cases were diagnosed and 8.2 million cancer deaths were established $^{(1)}$. The overall age-standardized cancer incidence rates vary three- to fivefold across different regions of the world ${ }^{(2)}$. These variations may be related to different modifiable risk and preventive factors among which diet may play a central role. It has been estimated that about one-third of all cancer could be avoidable by changes in eating habits ${ }^{(3)}$.

Foods of plant origin have received particular interest over the years as potential cancer-preventive components of a healthy diet. Fruit and vegetables contain a myriad of bioactive phytochemicals that, through various molecular mechanisms, show chemopreventive properties in both in vitro and in vivo models of carcinogenesis ${ }^{(4)}$. However, from an epidemiological point of view, while early data from case-control studies suggested a clear preventive role for fruit and vegetables on cancer in different sites, recent large prospective studies have questioned this conclusion $^{(5)}$. Indeed, while an expert panel report from the World Cancer Research Fund/American Institute for Cancer Research published in 1997 stated that there was 'convincing' evidence that a high intake of fruit and/or 
vegetables prevents cancers, an updated report published 10 years later downgraded the evidence to either 'probable' or 'limited-suggestive,(6).

Because of the peculiar chemical composition and the potential molecular mechanisms involved, it is possible that some types of fruit/vegetable may be much more strongly associated with cancer risk than others. This may be hidden in epidemiological studies examining the association of cancer risk with total fruit/vegetable intake. In this respect, our interest was attracted by apples considering that they are the most consumed fruit in European countries and they are a rich source of bioactive phytochemicals (phenols and flavonoids) possessing strong chemopreventive and antioxidant activities ${ }^{(7)}$. We therefore conducted a systematic review of the literature on the relationship between apple intake and cancer risk, and for the first time undertook a meta-analysis to provide quantitative estimates of the association.

\section{Materials and methods}

\section{Literature search strategy}

We carried out a comprehensive literature search, without restrictions, up to December 2015 through PubMed (http:// www.ncbi.nlm.nih.gov/pubmed/), Web of Science (http:// wokinfo.com/) and Embase (http://www.embase.com) to identify all original articles on the association between apple intake and cancer risk using the following search keywords: (apple OR apples OR Rosaceae OR Malus domestica) AND (cancer OR neoplastic disease OR neoplasm). Furthermore, the reference lists of included articles and recent relevant reviews were manually examined to identify additional relevant publications. The standard procedures for conducting and reporting meta-analysis according to the guidelines from the Meta-analysis Of Observational Studies in Epidemiology (MOOSE) group were followed ${ }^{(8)}$.

\section{Selection criteria}

Potential identified articles were included if they met the following criteria: (i) used a case-control or prospective study design; (ii) evaluated the association between apple intake and cancer risk; and (iii) presented OR, relative risk (RR) or hazard ratio (HR) estimates with 95\% CI. When there were several publications from the same study, the publication with the largest number of cases was selected. Although useful to have background information, reviews and meta-analysis were excluded.

\section{Data abstraction and quality assessment}

For each potential study included, two investigators independently carried out the selection evaluation, data abstraction and quality assessment; disagreements between evaluators concerning the selected studies were resolved by discussion or in consultation with a third author. From the selected studies we extracted the following data: the first author's last name, year of publication, study region and design, tumour site, sample size (number of cases and controls; cohort size and incident cases), age, duration of follow-up for cohort studies, dietary assessment method, apple intake and OR/RR/HR estimates with 95\% CI for the highest $v$. lowest category of apple intake. When multiple estimates were reported in the article, we abstracted those that adjusted for the most confounding factors. If separate risk estimates for males and females were available in one study, we treated them as two separate studies.

The study quality was assessed by a nine-star system based on the Newcastle-Ottawa Scale method ${ }^{(9)}$. The full score was 9 and a total score $\geq 7$ was used to indicate a high-quality study. To avoid selection bias, no study was excluded because of these quality criteria.

\section{Statistical analysis}

For the overall estimation, the HR and RR were taken to be approximations to $\mathrm{OR}$, and the meta-analysis was done as if all types of ratio were OR. The combined risk estimates were calculated using the random-effects model.

The $\chi^{2}$-based Cochran's $Q$ statistic and the $I^{2}$ statistic were used to evaluate heterogeneity in results across studies. For the $Q$ statistic, a $P$ value $<0 \cdot 1$ was considered to be representative of statistically significant heterogeneity. The $I^{2}$ statistic yields results ranging from 0 to $100 \%$ $\left(I^{2}=0-25 \%\right.$, absent; $I^{2}=25-50 \%$, low; $I^{2}=50-75 \%$, moderate; and $I^{2}=75-100 \%$, high heterogeneity).

Analysis of publication bias was carried out by both Egger's linear regression test and Begg's rank correlation test. Both methods test for funnel plot asymmetry, the former being based on the rank correlation between the effect estimates and their sampling variances, and the latter on a linear regression of a standard normal deviate on its precision. If a potential bias was detected, we further conducted a sensitivity analysis to assess the robustness of combined effect estimates and the possible influence of the bias and to have the bias corrected. A sensitivity analysis was conducted to investigate the influence of a single study on the risk estimate by omitting each study in turn. Funnel plots were considered asymmetric if the intercept of Egger's regression line deviated from zero with a $P$ value of $<0 \cdot 05$. The ProMeta Version $2 \cdot 0$ statistical program (Internovi) was used for the analysis. All reported $P$ values are from two-sided statistical tests and differences with $P \leq 0.05$ were considered significant.

\section{Results}

\section{Literature search}

As shown in Fig. 1, 2923 articles were obtained by searching the three different databases (PubMed, Web of Science and Embase). After excluding 997 duplicates, 1926 records were identified for title and abstract revision. Of 




Fig. 1 Flowchart of the selection process for inclusion of studies in the meta-analysis

the 1926 articles screened, 1883 were excluded because they were not observational studies, leaving forty-three articles for full-text revision. Hand searching of reference lists of both selected articles and recent relevant reviews led to the identification of eight additional items. Ten papers were subsequently excluded because they did not met the inclusion criteria as follows: two studies did not report the amount of apple intake; three articles reported the same data of a previous publication; two publications (lowest case numbers) were from the same study; one study did not report the risk estimate and another did not report the CI; and one evaluated the cancer risk in association with apple juice and cider. Therefore, at the end of the selection process, forty-one studies met the inclusion criteria and were included in the systematic review and meta-analysis ${ }^{(10-50)}$.

\section{Study characteristics and quality assessment}

Of the forty-one selected papers, twenty were casecontrol studies ${ }^{(10-29)}$, seventeen were cohort studies ${ }^{(30-46)}$ and four were pooled analyses of cohort studies ${ }^{(47-50)}$. General characteristics of case-control and cohort studies are shown in Tables 1 and 2, respectively.

Case-control studies were published between 1994 and 2014; three were conducted in Italy ${ }^{(13,19,22)}$, two each in $\operatorname{Iran}^{(10,15)}$, China ${ }^{(12,23)}$ and Australia ${ }^{(14,29)}$, and one each in Poland ${ }^{(16)}$, Spain ${ }^{(11)}$, Czech Republic ${ }^{(17)}$, Brazil ${ }^{(18)}$, $\mathrm{UK}^{(20)}$, India ${ }^{(24)}$, Hawaii ${ }^{(25)}$, Mexico ${ }^{(26)}$, Sweden ${ }^{(27)}$ and Uruguay $^{(28)}$. One was a multinational study conducted in nine countries worldwide (Italy, Spain, Poland, Northern Ireland, India, Cuba, Canada, Australia and Sudan) ${ }^{(21)}$. Three case-control studies were on lung cancer (2049 cases and 4044 controls), five on colorectal cancer (3319 cases and 10158 controls), five on breast cancer (7682 cases and 11880 controls), two on oesophageal cancer (447 cases and 6725 controls), three on oral cavity cancer (2859 cases and 8943 controls), two on prostate cancer (1344 cases and; 6729 controls) and one each on pancreas, bladder, larynx, ovary, kidney and brain (glioma) cancer. 


\begin{tabular}{|c|c|c|c|c|c|c|}
\hline $\begin{array}{l}\text { First author, year, reference, } \\
\text { location }\end{array}$ & No. of cases/controls (age) & $\begin{array}{l}\text { Dietary } \\
\text { assessment }\end{array}$ & Apple intake comparisons & OR & $95 \% \mathrm{Cl}$ & $\begin{array}{l}\text { Confounding factors adjusted } \\
\text { for }\end{array}$ \\
\hline \multicolumn{7}{|l|}{ Lung cancer $n 3$} \\
\hline $\begin{array}{l}\text { Tarrazzo-Antelo }(2014)^{(11)} \text {, } \\
\text { Spain }\end{array}$ & $\begin{array}{l}371 \text { (median } 69 \text { years)/ } \\
496 \text { (median } 63 \text { years) }\end{array}$ & FFQ & 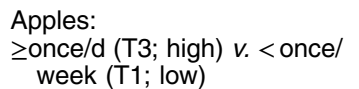 & 0.75 & $0.49,1.15$ & Age, sex, smoking \\
\hline $\begin{array}{l}\text { Kubik (2008) })^{(17)} \text {, Czech } \\
\text { Republic }\end{array}$ & $\begin{array}{l}509 \text { males, } 587 \text { females/ } \\
788 \text { males, } 2178 \text { females } \\
\text { ( } 25-89 \text { years) }\end{array}$ & $\begin{array}{l}\mathrm{FFQ} \\
9 \text { food items }\end{array}$ & $\begin{array}{l}\text { Apples: } \\
\text { Daily or several times per } \\
\quad \text { week (high) } v \text {. never (low) }\end{array}$ & $\begin{array}{l}\text { Females: } \\
\text { Current smokers } 0.77 \\
\text { Never smokers } 0.93 \\
\text { Males: }\end{array}$ & $\begin{array}{l}0.56,1.04 \\
0.61,1.41\end{array}$ & $\begin{array}{l}\text { Age, residence, education, } \\
\text { smoking }\end{array}$ \\
\hline $\begin{array}{l}\text { Le Marchand }(2000)^{(25)} \\
\text { Hawaii, USA }\end{array}$ & $\begin{array}{l}375 \text { males (mean } 65.5 \text { years), } \\
207 \text { females (mean } 66.0 \text { years)/ } \\
375 \text { males (mean } 65.4 \text { years), } \\
207 \text { females (mean } 65.6 \text { years) }\end{array}$ & $\begin{array}{l}\text { Validated FFQ } \\
242 \text { food items }\end{array}$ & $\begin{array}{l}\text { Apples, g/d: } \\
>49 \cdot 7 \text { (Q4; high) v. <2.3 (Q1; } \\
\text { low) }\end{array}$ & $\begin{array}{c}\text { Current smokers } 0.77 \\
\text { Never smokers } 0.54 \\
0.6\end{array}$ & $\begin{array}{c}0.54,1.10 \\
0.29,1.09 \\
0.4,1.0\end{array}$ & $\begin{array}{l}\text { Age, sex, ethnicity, smoking, } \\
\beta \text {-carotene, saturated fat }\end{array}$ \\
\hline \multicolumn{7}{|l|}{ Colorectal cancer $(n 5)$} \\
\hline $\begin{array}{l}\text { Annema (2011) } \\
\text { Western Australia }\end{array}$ & $\begin{array}{l}918(40-79, \text { mean } 64.9(\mathrm{sD} 8.9) \text { years)/ } \\
1021 \text { (mean } 64.6(\mathrm{sD} 9.4) \text { years) }\end{array}$ & $\begin{array}{l}\mathrm{FFQ} \\
74 \text { food items }\end{array}$ & $\begin{array}{l}\text { Apples, servings/d: } \\
\geq 0.50 \text { (Q4; high) v. }<0.07 \\
\text { (Q1; low) }\end{array}$ & 0.74 & $0.56,0.99$ & $\begin{array}{l}\text { Age, sex, BMl at age } 20 \\
\text { years, El, multivitamin, } \\
\text { alcohol, PA, smoking, } \\
\text { diabetes, SES }\end{array}$ \\
\hline $\begin{array}{l}\text { Jedrychowski }(2010)^{(16)} \\
\quad \text { Poland }\end{array}$ & $\begin{array}{l}592 / 765 \\
\mathrm{~N} / \mathrm{A}\end{array}$ & $\begin{array}{l}\text { EPIC-FFQ } \\
148 \text { food items }\end{array}$ & $\begin{array}{l}\text { Apples, servings/d: } \\
>1.5 \text { (Q5; high) v. <0.18 (Q1; } \\
\text { low) }\end{array}$ & 0.53 & $0.35,0.79$ & $\begin{array}{l}\text { Age, sex, place of residency, } \\
\text { marital status, smoking, } \\
\text { total El, intake of } \\
\text { vegetables, intake of fruits }\end{array}$ \\
\hline $\begin{array}{l}\text { Theodoratou }(2007)^{(20)} \text {, } \\
\text { UK }\end{array}$ & $\begin{array}{l}1456 \text { (mean } 63.9 \text { (sD 9.6) years)/ } \\
1456 \text { (mean } 64.7 \text { (sD 9.5) years) }\end{array}$ & $\begin{array}{l}\text { Validated FFQ } \\
150 \text { food items }\end{array}$ & $\begin{array}{l}\text { Apples: } \\
\text { Q4 (high) v. Q1 (low) }\end{array}$ & 0.96 & $0.62,1.50$ & $\begin{array}{l}\text { Age, sex, residence area, } \\
\text { family history of CRC, total } \\
\text { El, fibre, alcohol, NSAID, } \\
\text { smoking, BMI, PA }\end{array}$ \\
\hline Gallus (2005) $)^{(22)}$, Italy & $\begin{array}{l}193 / 6629 \\
N / A\end{array}$ & $\begin{array}{l}\text { FFQ } \\
78 \text { food items }\end{array}$ & $\begin{array}{l}\text { Apples, portions/d: } \\
\geq 1 \text { (high) } v .<1 \text { (low) }\end{array}$ & 0.70 & $0.62,0.79$ & $\begin{array}{l}\text { Age, sex, study centre, } \\
\text { education, BMI, smoking, } \\
\text { alcohol, total El, vegetable } \\
\text { consumption, PA, other } \\
\text { fruit intake }\end{array}$ \\
\hline $\begin{array}{l}\text { Deneo-Pellegrini } \\
(1996)^{(28)} \text {, Uruguay }\end{array}$ & $\begin{array}{l}160 \text { (mean } 64.7 \text { years)/ } \\
287 \text { (mean } 65.3 \text { years) }\end{array}$ & $\begin{array}{l}\text { FFQ } \\
50 \text { food items }\end{array}$ & $\begin{array}{l}\text { Apples: } \\
\text { T3 (high) v. T1 (low) }\end{array}$ & 0.40 & $0.25,0.66$ & $\begin{array}{l}\text { Age, sex, residence, BMI, } \\
\text { total El, alcohol }\end{array}$ \\
\hline \multicolumn{7}{|l|}{ Breast cancer $(n 5)$} \\
\hline Bao (2012) ${ }^{(12)}$, China & $\begin{array}{l}3423 / 3464 \\
\text { (25-70 years) }\end{array}$ & $\begin{array}{l}\text { Validated FFQ } \\
76 \text { food items }\end{array}$ & $\begin{array}{l}\text { Rosaceae (apples, pears } \\
\text { and peaches), g/d: } \\
\geq 91.13(\mathrm{Q} 5 ; \text { high) v. }<12.51 \\
\text { (Q1; low) }\end{array}$ & 0.84 & $0.71,0.98$ & $\begin{array}{l}\text { Age, education, family } \\
\text { history of breast cancer, } \\
\text { history of breast fibro- } \\
\text { adenoma, exercise, BMI, } \\
\text { age at menarche, } \\
\text { menopausal status, total } \\
\text { meat, total fruit, total } \\
\text { vegetables }\end{array}$ \\
\hline Di Pietro $(2007)^{(18)}$, Brazil & $\begin{array}{l}33 / 33 \\
(30-70 \text { years })\end{array}$ & $\begin{array}{l}\text { FFQ } \\
91 \text { food items }\end{array}$ & $\begin{array}{l}\text { Apples: } \\
\text { weekly (high) v. never/rarely } \\
\text { (low) }\end{array}$ & 0.30 & $0.09,0.94$ & Family income \\
\hline Gallus (2005) $)^{(22)}$, Italy & $\begin{array}{l}2569 / 6629 \\
N / A\end{array}$ & $\begin{array}{l}\text { FFQ } \\
78 \text { food items }\end{array}$ & $\begin{array}{l}\text { Apples, portions/d: } \\
\geq 1 \text { (high) } v .<1 \text { (low) }\end{array}$ & 0.76 & $0.67,0.85$ & $\begin{array}{l}\text { Age, sex, study centre, } \\
\text { education, BMl, smoking, } \\
\text { alcohol, total El, vegetable } \\
\text { consumption, PA, other } \\
\text { fruit intake }\end{array}$ \\
\hline
\end{tabular}




\begin{tabular}{|c|c|c|c|c|c|c|}
\hline $\begin{array}{l}\text { First author, year, reference, } \\
\text { location }\end{array}$ & No. of cases/controls (age) & $\begin{array}{l}\text { Dietary } \\
\text { assessment }\end{array}$ & Apple intake comparisons & OR & $95 \% \mathrm{Cl}$ & $\begin{array}{l}\text { Confounding factors adjusted } \\
\text { for }\end{array}$ \\
\hline Malin $(2003)^{(23)}$, China & $\begin{array}{l}1459(47.8(\mathrm{sD} 8 \cdot 0) \text { years }) / \\
1556(47.2(\mathrm{sD} 8 \cdot 8) \text { years })\end{array}$ & $\begin{array}{l}\text { Validated FFQ } \\
76 \text { food items }\end{array}$ & $\begin{array}{l}\text { Apples, g/d: } \\
>57.0(\mathrm{Q} 5 ; \text { high) v. <3.2 (Q1; } \\
\text { low) }\end{array}$ & 0.86 & $0.66,1.11$ & $\begin{array}{l}\text { Age, education, family } \\
\text { history of breast cancer } \\
\text { and fibro-adenoma, WHR, } \\
\text { menarche age, PA, ever } \\
\text { had live birth, age at first } \\
\text { live birth, total El }\end{array}$ \\
\hline $\begin{array}{l}\text { Torres-Sánchez }(2000)^{(26)} \text {, } \\
\text { Mexico }\end{array}$ & $\begin{array}{l}\text { 198/198 } \\
\text { (29-71 years) }\end{array}$ & $\begin{array}{l}\text { Validated FFQ } \\
95 \text { food items }\end{array}$ & $\begin{array}{l}\text { Apples, pieces/week: } \\
\geq 1 \text { (high) } v .<1 \text { (low) }\end{array}$ & 0.83 & $0.54,1.28$ & $\begin{array}{l}\text { Age, total El, age at } \\
\text { menarche, number of } \\
\text { children, age at first birth, } \\
\text { lifetime lactation, family } \\
\text { history of breast cancer }\end{array}$ \\
\hline \multicolumn{7}{|l|}{ Oesophageal cancer $(n 2)$} \\
\hline Hajizadeh $(2011)^{(15)}$, Iran & $\begin{array}{l}47 \text { (40-75, mean } 58(\mathrm{sD} \mathrm{10.1)} \mathrm{years)/} \\
96 \text { (mean } 58 \text { (sD 10.4) years) }\end{array}$ & $\begin{array}{l}\text { Validated FFQ } \\
168 \text { food items }\end{array}$ & $\begin{array}{l}\text { Apples: } \\
\text { T3 (high) v. T1 (low) }\end{array}$ & 0.33 & $0.04,1.10$ & $\begin{array}{l}\text { Age, sex, education, } \\
\text { smoking, BMl, } \\
\text { symptomatic gastro- } \\
\text { oesophageal reflux, } \\
\text { total El }\end{array}$ \\
\hline Gallus $(2005)^{(22)}$, Italy & $\begin{array}{l}304 / 6629 \\
\text { N/A }\end{array}$ & $\begin{array}{l}\text { FFQ } \\
78 \text { food items }\end{array}$ & $\begin{array}{l}\text { Apples, portions/d: } \\
\geq 1 \text { (high) } v .<1 \text { (low) }\end{array}$ & 0.78 & $0.56,1.09$ & $\begin{array}{l}\text { Age, sex, study centre, } \\
\text { education, BMl, smoking, } \\
\text { alcohol, total El, vegetable } \\
\text { consumption, PA, other } \\
\text { fruit intake }\end{array}$ \\
\hline \multicolumn{7}{|l|}{ Pancreatic cancer } \\
\hline Rossi $(2012)^{(13)}$, Italy & $\begin{array}{l}326 / 652 \\
\text { (34-80, median } 63 \text { years) }\end{array}$ & $\begin{array}{l}\text { Validated FFQ } \\
78 \text { food items }\end{array}$ & $\begin{array}{l}\text { Apples and pears, portions/d: } \\
\geq 3 \text { (Q5; high) v. <0.5 (Q1; } \\
\text { low) }\end{array}$ & 0.35 & $0.15,0.82$ & $\begin{array}{l}\text { Age, sex, study centre, year } \\
\text { of interview, education, } \\
\text { history of diabetes, } \\
\text { smoking, alcohol, non- } \\
\text { alcohol El }\end{array}$ \\
\hline \multicolumn{7}{|l|}{ Bladder cancer } \\
\hline Sacerdote $(2007)^{(19)}$, Italy & $\begin{array}{l}266 / 193 \\
\text { (40-75 years) }\end{array}$ & $\begin{array}{l}\text { FFQ } \\
22 \text { food items }\end{array}$ & $\begin{array}{l}\text { Apples: } \\
>\text { median (high) } v . \leq \text { median } \\
\quad \text { (low) }\end{array}$ & 0.63 & $0.39,0.99$ & $\begin{array}{l}\text { Age, smoking status and } \\
\text { maximum number of } \\
\text { cigarettes }\end{array}$ \\
\hline \multicolumn{7}{|l|}{ Oral cavity cancer $(n 3)$} \\
\hline $\begin{array}{l}\text { Poland, Northern } \\
\text { Ireland, India, Cuba, } \\
\text { Canada, Australia and } \\
\text { Sudan) }\end{array}$ & $\mathrm{N} / \mathrm{A}$ & 25 food items & Q4 (high) v. Q1 (low) & 0.4 & $0.3,0.5$ & $\begin{array}{l}\text { tobacco smoking, tobacco } \\
\text { chewing, alcohol, BMI, } \\
\text { total number of portions } \\
\text { per week }\end{array}$ \\
\hline Gallus $(2005)^{(22)}$, Italy & $\begin{array}{l}598 / 6629 \\
\text { N/A }\end{array}$ & $\begin{array}{l}\mathrm{FFQ} \\
78 \text { food items }\end{array}$ & $\begin{array}{l}\text { Apples, portions/d: } \\
\geq 1 \text { (high) } v .<1 \text { (low) }\end{array}$ & 0.82 & $0.65,1.05$ & $\begin{array}{l}\text { Age, sex, study centre, } \\
\text { education, BMl, smoking, } \\
\text { alcohol, total El, vegetable } \\
\text { consumption, PA, other } \\
\text { fruit intake }\end{array}$ \\
\hline $\begin{array}{l}\text { Rajkumar }(2003)^{(24)} \\
\text { Southern India }\end{array}$ & $\begin{array}{l}309 \text { males (22-85, median } 56 \text { years), } \\
282 \text { females (18-87, median } 58 \\
\text { years) } \\
292 \text { males ( } 20-76, \text { median } 55 \text { years), } \\
290 \text { females (18-80, median } 52 \\
\text { years) }\end{array}$ & $\begin{array}{l}\mathrm{FFQ} \\
21 \text { food items }\end{array}$ & $\begin{array}{l}\text { Apples or pears, servings/ } \\
\text { week: } \\
\geq 1 \text { (T3; high) v. } 0 \text { ( } \mathrm{T} 1 \text {; low) }\end{array}$ & 0.04 & $0.02,0.08$ & $\begin{array}{l}\text { Age, sex, centre, education, } \\
\text { chewing, smoking, } \\
\text { drinking habits }\end{array}$ \\
\hline
\end{tabular}




\section{Table 1 Continued}

\begin{tabular}{|c|c|c|c|c|c|c|}
\hline $\begin{array}{l}\text { First author, year, reference, } \\
\text { location }\end{array}$ & No. of cases/controls (age) & $\begin{array}{l}\text { Dietary } \\
\text { assessment }\end{array}$ & Apple intake comparisons & OR & $95 \% \mathrm{Cl}$ & $\begin{array}{l}\text { Confounding factors adjusted } \\
\text { for }\end{array}$ \\
\hline \multicolumn{7}{|l|}{ Larynx cancer } \\
\hline Gallus $(2005)^{(22)}$, Italy & $\begin{array}{l}460 / 6629 \\
\text { N/A }\end{array}$ & $\begin{array}{l}\text { FFQ } \\
78 \text { food items }\end{array}$ & $\begin{array}{l}\text { Apples, portions/d: } \\
\geq 1 \text { (high) } v .<1 \text { (low) }\end{array}$ & 0.59 & $0.45,0.78$ & $\begin{array}{l}\text { Age, sex, study centre, } \\
\text { education, BMI, smoking, } \\
\text { alcohol, total El, vegetable } \\
\text { consumption, PA, other } \\
\text { fruit intake }\end{array}$ \\
\hline \multicolumn{7}{|l|}{ Ovarian cancer } \\
\hline Gallus $(2005)^{(22)}$, Italy & $\begin{array}{l}1031 / 6629 \\
\text { N/A }\end{array}$ & $\begin{array}{l}\text { FFQ } \\
78 \text { food items }\end{array}$ & $\begin{array}{l}\text { Apples, portions/d: } \\
\geq 1 \text { (high) } v .<1 \text { (low) }\end{array}$ & 0.76 & $0.65,0.90$ & $\begin{array}{l}\text { Age, sex, study centre, } \\
\text { education, BMI, smoking, } \\
\text { alcohol, total El, vegetable } \\
\text { consumption, PA, other } \\
\text { fruit intake }\end{array}$ \\
\hline \multicolumn{7}{|l|}{ Prostate cancer } \\
\hline Gallus $(2005)^{(22)}$, Italy & $\begin{array}{l}1294 / 6629 \\
\mathrm{~N} / \mathrm{A}\end{array}$ & $\begin{array}{l}\text { FFQ } \\
78 \text { food items }\end{array}$ & $\begin{array}{l}\text { Apples, portions/d: } \\
\geq 1 \text { (high) } v .<1 \text { (low) }\end{array}$ & 0.93 & $0.79,1.10$ & $\begin{array}{l}\text { Age, sex, study centre, } \\
\text { education, BMl, smoking, } \\
\text { alcohol, total El, vegetable } \\
\text { consumption, PA, other } \\
\text { fruit intake }\end{array}$ \\
\hline Askari $(2014)^{(10)}$, Iran & $\begin{array}{l}50 \text { (40-78 years)/ } \\
100 \text { (43-71 years) }\end{array}$ & $\begin{array}{l}\text { Validated FFQ } \\
168 \text { food items }\end{array}$ & $\begin{array}{l}\text { Apple, g/d: } \\
>56.41 \text { (T3; high) v. }<7.59 \\
(\mathrm{~T} 1 ; \text { low) }\end{array}$ & 0.60 & $0.02,1.03$ & Age, diabetes, smoking, total \\
\hline \multicolumn{7}{|l|}{ Kidney cancer } \\
\hline $\begin{array}{l}\text { Lindblad (1997)(27), } \\
\text { Sweden }\end{array}$ & $\begin{array}{l}379 \text { males (mean } 63.6 \text { years) and } \\
\text { females (mean } 64.4 \text { years)/ } \\
350 \text { males (mean } 62.7 \text { years) and } \\
\text { females (63.4 years) }\end{array}$ & $\begin{array}{l}\text { FFQ } \\
63 \text { food items }\end{array}$ & $\begin{array}{l}\text { Apples, g/week: } \\
>656 \text { (Q4; high) v. <105 (Q1; } \\
\text { low) }\end{array}$ & 0.65 & $0.43,0.98$ & $\begin{array}{l}\text { Age, sex, BMI, smoking, } \\
\text { educational level }\end{array}$ \\
\hline \multicolumn{7}{|l|}{ Brain cancer (glioma) } \\
\hline Giles $(1994)^{(29)}$, Australia & $\begin{array}{l}243 \text { males (mean } 50.0 \text { years), } 166 \\
\text { females (mean } 48.8 \text { years) } / \\
243 \text { males (mean } 50.8 \text { years), } 166 \\
\text { females (mean } 49.9 \text { years) }\end{array}$ & $\begin{array}{l}\text { FFQ } \\
59 \text { food items }\end{array}$ & $\begin{array}{l}\text { Apples: } \\
\text { Q4 (high) v. Q1 (low) }\end{array}$ & $\begin{array}{l}\text { Males } 2.04 \\
\text { Females } 1.00\end{array}$ & $\begin{array}{l}1 \cdot 04,4 \cdot 00 \\
0.25,4.04\end{array}$ & Age, alcohol, smoking \\
\hline
\end{tabular}


drugs; WHR, waist-to-hip ratio. 
Table 2 Characteristics of cohort studies on apple consumption in association with various types of cancer included in the systematic review

\begin{tabular}{|c|c|c|c|c|c|c|}
\hline $\begin{array}{l}\text { First author, year, reference, } \\
\text { location }\end{array}$ & $\begin{array}{l}\text { No. of subjects, (age), } \\
\text { follow-up }\end{array}$ & Dietary assessment & Apple intake comparisons & HR/RR & $95 \% \mathrm{Cl}$ & $\begin{array}{l}\text { Confounding factors } \\
\text { adjusted for }\end{array}$ \\
\hline \multicolumn{7}{|l|}{ Lung cancer $(n 7)$} \\
\hline $\begin{array}{l}\text { Büchner }(2010)^{(32)} \text {, ten } \\
\text { European countries (EPIC) }\end{array}$ & $\begin{array}{l}478535 \text { cohort } \\
1830 \text { incident cases } \\
\text { (25-70 years) } \\
\text { Follow-up } 8.7 \text { years }\end{array}$ & Validated FFQ & $\begin{array}{l}\text { Apples and pears (hard fruit): } \\
\text { increments of } 25 \mathrm{~g} / \mathrm{d}\end{array}$ & $\begin{array}{l}\text { Full cohort } 0.99 \\
\text { Current smokers } 0.99\end{array}$ & $\begin{array}{l}0.96-1.02 \\
0.97,1.01\end{array}$ & $\begin{array}{l}\text { Age, sex, vegetable consumption, } \\
\text { smoking, El, weight, height, alcohol, } \\
\text { PA, school level }\end{array}$ \\
\hline \multirow[t]{3}{*}{ Wright $(2008)^{(36)}$, USA } & $\begin{array}{l}472081 \text { cohort } \\
6035 \text { incident cases } \\
\text { (50-71 years) } \\
\text { Follow-up } 8 \text { years }\end{array}$ & $\begin{array}{l}\text { Validated FFQ } \\
124 \text { food items }\end{array}$ & $\begin{array}{l}\text { Rosaceae*, servings } / 1000 \mathrm{kcal} \\
\text { per d: } \\
\text { Males } \\
0.72 \text { (Q5; high) v. } 0.003 \text { (Q1; low) } \\
\text { Females } \\
0.94 \text { (Q5; high) v. } 0.005 \text { (Q1; low) }\end{array}$ & $\begin{array}{l}\text { Males: } \\
\text { Full cohort } 0.82 \\
\text { Never smokers } 0.75 \\
\text { Former smokers } 0.81 \\
\text { Current smokers } 0.76 \\
\text { Females: }\end{array}$ & $\begin{array}{l}0.73,0.91 \\
0.45,1.26 \\
0.70,0.93 \\
0.62,0.94\end{array}$ & \multirow[t]{3}{*}{$\begin{array}{l}\text { Age, El, race, education, BMI, smoking } \\
\text { status, smoking dose, time since } \\
\text { quitting smoking, alcohol intake, PA, } \\
\text { family history of any cancer }\end{array}$} \\
\hline & & & & $\begin{array}{l}\text { Full cohort } 0.97 \\
\text { Never smokers } 1.31 \\
\text { Former smokers } 0.91\end{array}$ & $\begin{array}{l}0.85,1.12 \\
0.76,2.26 \\
0.73,1.13\end{array}$ & \\
\hline & & & & Current smokers 1.01 & $0.82,1.24$ & \\
\hline $\begin{array}{l}\text { Linseisen }(2007)^{(38)} \text {, ten } \\
\quad \text { European countries (EPIC) }\end{array}$ & $\begin{array}{l}478590 \text { cohort } \\
1126 \text { incident cases (608 } \\
\text { males, } 518 \text { females) } \\
\text { (25-70 years) } \\
\text { Follow-up } 6.4 \text { years }\end{array}$ & $\begin{array}{l}\text { Calibrated FFQ, } \\
24 \mathrm{~h} \text { diet recalls }\end{array}$ & $\begin{array}{l}\text { Apples and pears, g/d: } \\
115 \cdot 0-2269 \cdot 4 \text { (Q5; high) } \\
\text { v. 0-11.8 (Q1; low) }\end{array}$ & $\begin{array}{l}\text { Full cohort } 0.85 \\
\text { Never smokers } 0.95 \\
\text { Former smokers } 1.19 \\
\text { Current smokers } 0.80\end{array}$ & $\begin{array}{l}0.69,1.05 \\
0.41,2.22 \\
0.77,1.83 \\
0.62,1.04\end{array}$ & $\begin{array}{l}\text { Age, weight and height, red meat, } \\
\text { processed meat, alcohol, energy (fat } \\
\text { and non-fat), PA, education, } \\
\text { smoking }\end{array}$ \\
\hline Knekt $(2002)^{(42)}$, Finland & $\begin{array}{l}5218 \text { male cohort } \\
169 \text { incident cases } \\
\text { (mean } 39 \cdot 3 \text { (sD } 15 \cdot 8 \text { ) years) } \\
\text { Follow-up max. } 30 \text { years }\end{array}$ & $\begin{array}{l}\mathrm{FFQ} \\
>100 \text { food items }\end{array}$ & $\begin{array}{l}\text { Apples, g/d: } \\
>4.0 \text { (Q4; high) v. } 0.0 \text { (Q1; low) }\end{array}$ & 0.40 & $0.22,0.74$ & $\begin{array}{l}\text { Age, sex, geographic area, occupation, } \\
\text { smoking, BMl }\end{array}$ \\
\hline Arts $(2001)^{(43)}$, Netherlands & $\begin{array}{l}728 \text { cohort, male } \\
96 \text { incident cases } \\
\text { (65-84 years) } \\
\text { Follow-up } 10 \text { years }\end{array}$ & $\begin{array}{l}\text { Cross-check dietary } \\
\text { history method }\end{array}$ & $\begin{array}{l}7.5 \mathrm{mg} \text { catechin intake increase } \\
\text { from apples }\end{array}$ & 0.67 & $0.38,1 \cdot 17$ & $\begin{array}{l}\text { Age, other catechin sources, PA, total } \\
\text { El, alcohol, smoking, BMI, coffee, } \\
\text { fibre, vitamin C, vitamin E, } \beta- \\
\text { carotene }\end{array}$ \\
\hline $\begin{array}{l}\text { Feskanich }(2000)^{(45)}, \text { USA } \\
\quad(\mathrm{NHS})\end{array}$ & $\begin{array}{l}77283 \text { female cohort } \\
519 \text { incident cases } \\
\text { (30-55 years) } \\
\text { Follow-up } 12 \text { years }\end{array}$ & $\begin{array}{l}\text { Validated FFQ } \\
6 \text { fruit/23 vegetable } \\
\text { items }\end{array}$ & $\begin{array}{l}\text { Apples and pears: } \\
\text { increases of } 1 \text { serving } / \mathrm{d}\end{array}$ & 0.63 & $0.43,0.91$ & $\begin{array}{l}\text { Age, follow-up cycle, smoking status, } \\
\text { El, availability of diet data after } \\
\text { baseline measure }\end{array}$ \\
\hline $\begin{array}{l}\text { Smith-Warner }(2003)^{(48)} \\
\text { USA-Canada-Europe } \\
\text { Pooled analysis } \\
\text { Seven studies }\end{array}$ & $\begin{array}{l}262429 \text { female cohort } \\
137336 \text { male cohort } \\
3138 \text { incident cases } \\
\text { Follow-up 6-16 years }\end{array}$ & $\begin{array}{l}\text { Validated FFQ } \\
6-26 \text { fruit items }\end{array}$ & $\begin{array}{l}\text { Apples, pears, servings/d: } \\
\geq 0.5 \text { (Q4; high) } v .<0 \text { (Q1; low) }\end{array}$ & 0.80 & $0.68,0.94$ & $\begin{array}{l}\text { Education, BMI, alcohol intake, El, } \\
\text { smoking status }\end{array}$ \\
\hline \multicolumn{7}{|l|}{ Colorectal cancer ( $n$ 2) } \\
\hline $\begin{array}{l}\text { Lin }(2006)^{(39)} \text {, USA (NHS and } \\
\text { HPFS) }\end{array}$ & $\begin{array}{l}71976 \text { female cohort } \\
498 \text { incident cases ( } 30-55 \\
\text { years) } \\
35425 \text { male cohort } \\
380 \text { incident cases } \\
\text { (40-75 years) } \\
\text { Follow-up } 10 \text { years }\end{array}$ & $\begin{array}{l}\text { Validated FFQ } \\
131 \text { food items }\end{array}$ & $\begin{array}{l}\text { Apple: } \\
\geq 2 \text { servings/d (Q5; high) v. 0-2 } \\
\text { servings/week (Q1; low) }\end{array}$ & $\begin{array}{l}\text { NHS females } 0.64 \\
\text { HPFS males } 0.82\end{array}$ & $\begin{array}{l}0.35,1.17 \\
0.51,1.30\end{array}$ & $\begin{array}{l}\text { Age, BMI, PA, history of CRC, previous } \\
\text { colorectal polyps, prior screening } \\
\text { sigmoidoscopy or colonoscopy, } \\
\text { smoking, multivitamin use, current } \\
\text { aspirin use, alcohol, El, red meat, } \\
\text { total } \mathrm{Ca} \text {, total folate, total fibre }\end{array}$ \\
\hline $\begin{array}{l}\text { Koushik }(2007)^{(49)}, \text { USA- } \\
\text { Canada-Europe } \\
\text { Pooled analysis } \\
\text { Eleven studies }\end{array}$ & $\begin{array}{l}688904 \text { cohort (202 } 479 \\
\text { males, } 486425 \text { females) } \\
5489 \text { incident cases ( } 1651 \\
\text { males, 3838 females) } \\
\text { Follow-up 8-21 years }\end{array}$ & $\begin{array}{l}\text { Validated } \mathrm{FFQ} \\
4-21 \text { fruit items }\end{array}$ & $\begin{array}{l}\text { Apples, pears, applesauce, } \\
\text { servings/d: } \\
\geq 0.5 \text { (Q4; high) } v .<0 \text { (Q1; low) }\end{array}$ & 0.98 & $0.88,1 \cdot 10$ & $\begin{array}{l}\text { Age, BMI, height, education, PA, family } \\
\text { history of CRC, postmenopausal } \\
\text { hormone use, OC use, use of } \\
\text { NSAID, multivitamin use, smoking, } \\
\text { red meat, total milk, alcohol, El }\end{array}$ \\
\hline \multicolumn{7}{|l|}{ Breast cancer $(n 3)$} \\
\hline Boggs $(2010)^{(30)}$, USA & $\begin{array}{l}51928 \text { female cohort } \\
1268 \text { incident cases } \\
\text { (21-69 years) } \\
\text { Follow-up } 12 \text { years }\end{array}$ & $\begin{array}{l}\text { Validated FFQ } \\
85 \text { food items }\end{array}$ & $\begin{array}{l}\text { Apples: } \\
\geq 3 \text { servings/week (Q4; high) v. } \\
\quad \text { <1 serving/month (Q1; low) }\end{array}$ & 1.02 & $0.83,1.25$ & $\begin{array}{l}\text { Age, El, age at menarche, BMl at age } \\
18 \text { years, family history of breast } \\
\text { cancer, education, geographic } \\
\text { region, parity, age at first birth, OC } \\
\text { use, menopausal status, age at } \\
\text { menopause, menopausal hormone } \\
\text { use, vigorous activity, smoking, } \\
\text { alcohol, multivitamin use }\end{array}$ \\
\hline
\end{tabular}




\begin{tabular}{|c|c|c|c|c|c|c|}
\hline $\begin{array}{l}\text { First author, year, reference, } \\
\text { location }\end{array}$ & $\begin{array}{l}\text { No. of subjects, (age), } \\
\text { follow-up }\end{array}$ & Dietary assessment & Apple intake comparisons & $\mathrm{HR} / \mathrm{RR}$ & $95 \% \mathrm{Cl}$ & $\begin{array}{l}\text { Confounding factors } \\
\text { adjusted for }\end{array}$ \\
\hline $\begin{array}{l}\text { Adebamowo }(2005)^{(40)} \text {, USA } \\
\text { (NHS) }\end{array}$ & $\begin{array}{l}90638 \text { female cohort } \\
710 \text { incident cases } \\
\text { (25-46 years) } \\
\text { Follow-up } 8 \text { years }\end{array}$ & $\begin{array}{l}\text { Validated FFQ } \\
133-142 \text { items }\end{array}$ & $\begin{array}{l}\text { Apples: } \\
1 \text { servings/d (S6; high) } v .<1 \\
\text { servings/month (S1; low) }\end{array}$ & 1.16 & $0.77,1.76$ & $\begin{array}{l}\text { Age at menarche, parity, age at first } \\
\text { birth, family history of breast cancer } \\
\text { and benign breast disease, OC use, } \\
\text { alcohol, BMl, El, smoking, PA, } \\
\text { menopausal status }\end{array}$ \\
\hline $\begin{array}{l}\text { Smith-Warner }(2001)^{(47)} \\
\text { USA-Canada-Europe } \\
\text { Pooled analysis } \\
\text { Seven studies }\end{array}$ & $\begin{array}{l}336653 \text { female cohort } \\
7217 \text { incident cases } \\
\text { Follow-up 6-11 years }\end{array}$ & $\begin{array}{l}\text { Validated } \mathrm{FFQ} \\
4-21 \text { fruit items }\end{array}$ & $\begin{array}{l}\text { Apple, pears: } \\
100 \mathrm{~g} / \mathrm{d} \text { intake increment }\end{array}$ & 0.97 & $0.93,1.01$ & $\begin{array}{l}\text { Age at menarche, interaction between } \\
\text { parity and age at birth of first child, } \\
\text { OC use, history of benign breast } \\
\text { disease, menopausal status, } \\
\text { postmenopausal hormone use, } \\
\text { family history of breast cancer, } \\
\text { smoking, education, BMI, height, } \\
\text { alcohol, El }\end{array}$ \\
\hline $\begin{array}{l}\text { Oesophageal cancer } \\
\text { Freedman }(2007)^{(37)} \text {, USA }\end{array}$ & $\begin{array}{l}490802 \text { cohort }(292898 \\
\text { males, } 197904 \text { females) } \\
316 \text { incident cases }(103 \\
\quad \text { ESCC, } 213 \text { EAC) } \\
\text { (>50 years) } \\
\text { Follow-up } 5 \text { years }\end{array}$ & $\begin{array}{l}\text { Validated FFQ } \\
124 \text { food items }\end{array}$ & $\begin{array}{l}\text { Rosaceae, servings } / 1000 \text { kcal: } \\
0.63 \text { (T3; high) v. } 0.06 \text { (T1; low) }\end{array}$ & $\begin{array}{l}\text { ESCC } 0.34 \\
\text { EAC } 0.99\end{array}$ & $\begin{array}{l}0.18,0.65 \\
0.70,1.39\end{array}$ & $\begin{array}{l}\text { Age, sex, education, BMI, alcohol, } \\
\text { smoking, PA, total EI }\end{array}$ \\
\hline $\begin{array}{l}\text { Pancreatic cancer } \\
\text { Koushik }(2007)^{(50)}, \text { USA- } \\
\text { Canada-Europe-Australia } \\
\text { Pooled analysis } \\
\text { Fourteen studies }\end{array}$ & $\begin{array}{l}862584 \text { cohort } \\
2185 \text { incident cases }\end{array}$ & $\begin{array}{l}\text { Validated FFQ } \\
5-23 \text { fruit items }\end{array}$ & $\begin{array}{l}\text { Apples, pears, applesauce: } \\
3 \text { servings/week intake } \\
\text { increment }\end{array}$ & 0.98 & $0.94,1.03$ & $\begin{array}{l}\text { Age, smoking, alcohol, history of } \\
\text { diabetes, BMI, height, El }\end{array}$ \\
\hline $\begin{array}{l}\text { Bladder cancer } \\
\text { Büchner }(2009)^{(33)} \text {, ten } \\
\text { European countries (EPIC) }\end{array}$ & $\begin{array}{l}478533 \text { cohort } \\
1015 \text { incident cases } \\
\text { (25-70 years) } \\
\text { Follow-up } 8.7 \text { years }\end{array}$ & Validated FFQ & $\begin{array}{l}\text { Apples and pears (hard fruit): } \\
\text { increments of } 25 \mathrm{~g} / \mathrm{d}\end{array}$ & 0.90 & $0.82,0.98$ & $\begin{array}{l}\text { Age, sex, smoking, El from fat and non- } \\
\text { fat sources, vegetable consumption }\end{array}$ \\
\hline $\begin{array}{l}\text { Kidney cancer } \\
\text { Rashidkhani }(2005)^{(41)} \text {, Sweden }\end{array}$ & $\begin{array}{l}61000 \text { female cohort } \\
122 \text { incident cases } \\
\text { Follow-up } 13.4 \text { years }\end{array}$ & $\begin{array}{l}\text { Validated FFQ } \\
\text { Sixty-seven items }\end{array}$ & $\begin{array}{l}\text { Apple, serving/d: } \\
\geq 1 \text { (T3; high) v. } 0 \text { (T1; low) }\end{array}$ & 0.66 & $0.36,1 \cdot 18$ & Age, BMI \\
\hline $\begin{array}{l}\text { Endometrial cancer } \\
\text { Kabat }(2010)^{(31)} \text {, USA }\end{array}$ & $\begin{array}{l}112088 \text { female cohort } \\
1142 \text { incident cases } \\
\text { (50-71 years) } \\
\text { Follow-up } 8 \text { years }\end{array}$ & $\begin{array}{l}\text { Validated FFQ } \\
124 \text { food items }\end{array}$ & $\begin{array}{l}\text { Rosaceae, servings } / 1000 \mathrm{kcal} \\
\text { per d: } \\
\geq 0.56 \text { (Q5; high) v. <0.07 (Q1; } \\
\text { low) }\end{array}$ & 1.14 & $0.94,1.39$ & $\begin{array}{l}\text { Age, race/ethnicity, education, age at } \\
\text { menarche, parity, hormone therapy, } \\
\text { age at menopause, BMI, smoking, } \\
\text { PA, total fat intake, EI }\end{array}$ \\
\hline $\begin{array}{l}\text { Head-neck cancer } \\
\text { Freedman }(2008)^{(35)} \text {, USA }\end{array}$ & $\begin{array}{l}490802 \text { cohort } \\
787 \text { incident cases } \\
\text { (50-71 years) } \\
\text { Follow-up } 4.5 \text { years }\end{array}$ & Validated FFQ & $\begin{array}{l}\text { Rosaceae, servings } / 1000 \mathrm{kcal} \\
\text { per d: } \\
0.6 \text { (high) v. } 0.1 \text { (low) }\end{array}$ & 0.60 & $0.49,0.73$ & $\begin{array}{l}\text { Age, sex, alcohol, BMI, smoking, } \\
\text { education, total El, usual activity } \\
\text { throughout the day, PA }\end{array}$ \\
\hline $\begin{array}{l}\text { Urothelial cancer } \\
\text { Zeegers }(2001)^{(44)} \text {, Netherlands }\end{array}$ & $\begin{array}{l}3123 \text { cohort } \\
619 \text { incident cases } \\
\text { (55-69 years) } \\
\text { Follow-up } 6 \cdot 3 \text { years }\end{array}$ & $\begin{array}{l}\text { Validated FFQ } \\
150 \text { items }\end{array}$ & $\begin{array}{l}\text { Apples and pears: } \\
\text { increases of } 25 \mathrm{~g} / \mathrm{d}\end{array}$ & 0.97 & $0.91,1.03$ & $\begin{array}{l}\text { Age, sex, smoking, total fruit } \\
\text { consumption, total vegetable } \\
\text { consumption }\end{array}$ \\
\hline $\begin{array}{l}\text { Stomach cancer } \\
\text { Botterweck }(1998)^{(46)} \\
\text { Netherlands }^{(4)}\end{array}$ & $\begin{array}{l}120852 \text { cohort } \\
282 \text { incident cases } \\
\text { (55-69 years) } \\
\text { Follow-up } 6.3 \text { years }\end{array}$ & $\begin{array}{l}\text { Validated FFQ } \\
150 \text { items }\end{array}$ & $\begin{array}{l}\text { Apples and pears, median } \\
\text { intake, g/d: } \\
232.0 \text { (Q5; high) v. } 0.0 \text { (Q1; low) }\end{array}$ & 0.76 & $0.47,1.23$ & $\begin{array}{l}\text { Age, sex, smoking, education, stomach } \\
\text { disorders, family history of stomach } \\
\text { cancer, total vegetable consumption }\end{array}$ \\
\hline
\end{tabular}


Cohort studies were published between 1998 and 2012; nine were conducted in the $\mathrm{USA}^{(30,31,34-37,39,40,45)}$ and eight were conducted in Europe ${ }^{(32,33,38,41-44,46)}$. Pooled analysis were conducted in the USA, Canada, Europe and Australia and included from seven to fourteen prospective studies $^{(47-50)}$. Seven cohort studies were on lung cancer (1912199 cohort and 12913 incident cases), two on colorectal cancer (796305 cohort and 6367 incident cases), three on breast cancer (479219 cohort and 9195 incident cases), and one each on oesophageal, pancreatic, bladder, kidney, endometrial, head-neck, urothelial, stomach and all types of cancer.

Study-specific quality scores are summarized in the supplementary material, Table S1 and Table S2 for casecontrol and cohort studies, respectively. The range of quality score was from 4 to 8 (median $=6$, mean $=6 \cdot 25$, $\mathrm{SD}=1 \cdot 20)$ and from 7 to 9 (median $=8, \quad$ mean $=8 \cdot 1$, $\mathrm{SD}=0.85$ ) for case-control and cohort studies, respectively. High-quality studies (i.e. those studies that had seven awarded stars) included eight case-control ${ }^{(10,13-15,20,22,23,26)}$ and all twenty-one cohort ${ }^{(30-50)}$ studies.

\section{Apple intake and lung cancer risk}

Using the random-effect model, we found that the high intake of apple was significantly associated with a reduced risk of lung cancer in both case-control (OR $=0.75 ; 95 \% \mathrm{CI}$ $0.63,0.88 ; P=0.001, I^{2}=0 \%$; Fig. $2($ a) $)$ and cohort $(\mathrm{RR}=$ 0.89; 95\% CI 0.84, 0.94; $P<0.001, I^{2}=68 \%$; Fig. 2(b)) studies. A pooled analysis performed by combining casecontrol and cohort studies resulted in a significant $12 \%$ reduction of lung cancer risk $(\mathrm{RR}=0 \cdot 88$; $95 \% \mathrm{CI}$ 0.83, 0.92; $\left.P<0.001, I^{2}=65 \%\right)$ and allowed stratification based on both the sex and smoking status of the subjects (Table 3 ). A significant reduction of lung cancer risk was observed in current smokers $(\mathrm{RR}=0.88 ; 95 \%$ CI $0.77,1.00 ; P<0.042$, $\left.I^{2}=62 \%\right)$ and in studies where smokers and non-smokers were considered together $(\mathrm{RR}=0.82 ; 95 \% \mathrm{CI} 0.72,0.92$; $P=0.001, I^{2}=76 \%$, while the effect was not statistically significant in never smokers (Table 3 ). When stratifying the studies according to sex, apple intake was found to be significantly associated with lung cancer risk in males (RR $=0.79 ; 95 \%$ CI $\left.0.73,0.85 ; P<0.001, I^{2}=3 \%\right)$ but not in females $\left(\mathrm{RR}=0.92 ; 95 \%\right.$ CI $\left.0 \cdot 82,1 \cdot 03 ; P=0 \cdot 146, I^{2}=26 \%\right)$.

\section{Apple intake and risk of digestive tract cancers}

A significant reduction of colorectal cancer risk, associated with apple intake, was observed in case-control (OR= 0.56; $95 \%$ CI 0.54, 0.81; $P<0.001, I^{2}=55 \%$; Fig. 3(a)) but not in cohort $(\mathrm{RR}=0.93 ; 95 \%$ CI $0.79,1.10 ; P=0.4$, $I^{2}=13 \%$; Fig. 3(b)) studies. Combining case-control and cohort studies together resulted in a significant preventive effect of apple intake on colorectal cancer (RR $=0.72 ; 95 \%$ CI $0.59,0.88 ; P=0.001, I^{2}=77 \%$; Table 3). No cohort studies were found on oral cavity cancer risk and apple intake; however, the analysis on case-control studies 
showed a significant reduction of risk $(\mathrm{OR}=0 \cdot 25 ; 95 \%$ CI $\left.0.08,0.77 ; P=0.015, I^{2}=97 \%\right)$ even if high heterogeneity was observed (Table 3 ). Instead, no preventive effect of apples was observed on oesophageal cancer risk both in case-control and cohort studies. The pooled analysis of digestive tract cancers (colorectal, oral cavity, oesophageal and stomach) indicated that apple intake was inversely associated with the risk in case-control studies (OR $=0.50 ; 95 \%$ CI 0.36, 0.69; $P<0.001, I^{2}=90 \%$ ) but not in the cohort studies $(\mathrm{RR}=0.79 ; 95 \%$ CI $0.61,1.01$; $\left.P=0.063, I^{2}=61 \%\right)$.

\section{Apple intake and breast cancer risk}

A significant reduction of risk was observed for breast cancer only in case-control $(\mathrm{OR}=0.79 ; 95 \% \mathrm{CI} 0.73,0.87$; $P<0.001, I^{2}=1 \%$; Fig. $\left.4(\mathrm{a})\right)$ but not in cohort $(\mathrm{RR}=0.97$; 95\% CI 0.94, 1.01; $P=0 \cdot 192, I^{2}=0 \%$; Fig. 4(b)) studies. Pooled analysis resulted in a slightly significant effect $\left(\mathrm{RR}=0.89 ; 95 \%\right.$ CI 0.79, 1.00; $P=0 \cdot 047, I^{2}=69 \%$; Table 3).

\section{Apple intake and cancer risk in other anatomical sites}

In the case of prostate cancer, two case-control studies were found useful for the analysis that showed no association with apple intake $(\mathrm{OR}=0.93$; $95 \%$ CI 0.79, 1.09; $P=0.369, I^{2}=0 \%$; Table 3 ). For other anatomical sites, the availability of a single study did not allow the analysis.

\section{Publication bias and sensitivity analysis}

No evidence of publication bias could be detected for risk of colorectal, oral cavity, oesophageal and breast cancer. For the lung and overall digestive tract cancers no publication bias was observed in case-control studies. On the other hand, there was some evidence for publication bias regarding the risk of lung cancer in cohort studies and in pooled analysis as shown by both the Egger's regression test and funnel plot asymmetry (not shown). However, no publication bias could be detected by Begg's rank correlation test (the details are shown in Table 3).

Sensitivity analyses investigating the influence of a single study on the lung cancer risk estimate suggested that the risk estimates were not substantially modified by any single study. The lung risk estimates ranged from 0.85 (95\% CI 0.78, $\left.0.91 ; P<0.001, I^{2}=36 \%\right)$ omitting the study of Büchner et $a{ }^{(32)}$ to 0.92 (95\% CI $\left.0.87,0.99 ; P=0.015, I^{2}=67 \%\right)$ omitting the study of Wright et al. ${ }^{(36)}$. Of note, omitting the study of Büchner et $a l .{ }^{(32)}$ resulted in the absence of publication bias as evidenced by both Egger's regression $(P=0.659)$ and Begg's rank correlation $(P=0.528)$ tests.

\section{Discussion}

To the best of our knowledge, the present meta-analysis is the first one investigating the association between apple consumption and cancer risk in different anatomical sites. Apples are a cheap fruit, easy to store and transport, abundantly present and marketed all year, and therefore are among the most popular fruits in the world. For these reasons, we wondered whether an apple a day keeps the oncologist away $^{(22)}$. It is important to underline that in some studies the consumption of apple was asked as a single item $^{(10,11,14-20,22,23,25-29,36,40-43)}$ while in others the assessment regarded Rosaceae $e^{(12,31,35-37)}$ and 'apples and pears' together ${ }^{(13,21,24,32,33,38,44-50)}$. It would have been interesting to make a stratified analysis as a function of apple intake assessment to highlight the extent to which it influenced the results, but due to the small number of studies this was not possible. Therefore, confounding effects by the intake of other fruits may not be excluded. In particular, when considering the confounding adjusting factors (reported in the last column of Tables 1 and 2) the consumption of other fruit was taken into account only in a few cases ${ }^{(12,16,22,36,44)}$. Due to the small number of studies recovered for each tumour site and to summarize the overall effect size, the data derived from case-control and cohort studies have also been combined. The pooled results indicated that apple consumption (comparisons between the highest and the lowest category) was significantly associated with lower risk of different cancer types. However, when separately analysed on the basis of study type (case-control $v$. cohort), we generally found that the effect size was more consistent in case-control studies compared with cohort studies. In many cases the analysis of cohort studies did not evidence a significant effect of apple intake on cancer risk, while case-control studies showed a preventive effect. In particular, in the case of lung cancer, the reduction of risk associated with apple intake was statistically highly significant in both case-control and cohort studies. Instead, a significant preventive effect of apples on colorectal, breast and overall digestive tract cancers was found only in case-control studies. Similarly to our finding, a previous meta-analysis reported that prospective studies provide weaker evidence than case-control studies on the association of fruit and vegetable consumption with reduced cancer risk $^{(51)}$. It is common in meta-analysis to find higher effect size in case-control studies compared with cohort data $^{(52)}$. In general, case-control studies have several weaknesses and critical points which can lead to an overestimation of the effect. They can be affected by recall and selection bias, producing misclassification of exposure between case and control groups, and the control group may not be representative of the general population. On the other hand, it should be also considered that dietary assessment questionnaires used in prospective studies may be somewhat less accurate than those used in retrospective case-control settings. In the current meta-analysis we also found that case-control studies had lower median quality score than prospective studies (6 $v$. 8), so suggesting that findings derived from 
(a)



(b)

\begin{tabular}{|c|c|c|c|}
\hline & ES & $95 \% \mathrm{Cl}$ & Weight ( \\
\hline Wright $(2008)^{(36)} /$ female/never smokers & $1 \cdot 31$ & $0 \cdot 76,2 \cdot 26$ & 0.99 \\
\hline Linseisen $(2007)^{(38)} /$ female+male/former smokers & $1 \cdot 19$ & $0.77,1 \cdot 83$ & 1.52 \\
\hline Wright $(2008)^{(36)} /$ female/current smokers & 1.01 & $0.82,1 \cdot 24$ & $5 \cdot 16$ \\
\hline Büchner $(2010)^{(32)} / \mathrm{female}+$ male/current smokers & 0.99 & $0.97,1.01$ & $17 \cdot 30$ \\
\hline Büchner $(2010)^{(32)} /$ female+male/full cohort & 0.99 & $0.96,1.02$ & $16 \cdot 82$ \\
\hline Wright $(2008)^{(36)} /$ female/full cohort & 0.97 & $0 \cdot 85,1 \cdot 12$ & 8.51 \\
\hline Linseisen $(2007)^{(38)} /$ female+male/never smokers & 0.95 & $0.41,2 \cdot 22$ & 0.43 \\
\hline Wright $(2008)^{(36)} /$ female/former smokers & 0.91 & $0 \cdot 73,1 \cdot 13$ & $4 \cdot 77$ \\
\hline Linseisen $(2007)^{(38)} /$ female+male/full cohort & 0.85 & $0.69,1.05$ & 5.05 \\
\hline Wright $(2008)^{(36)} /$ male/full cohort & 0.82 & $0.73,0.91$ & $10 \cdot 47$ \\
\hline Wright $(2008)^{(36)} /$ male/former smokers & 0.81 & $0.70,0.93$ & $8 \cdot 25$ \\
\hline inseisen $(2007)^{(38)} /$ female+male/current smokers & 0.80 & $0.62,1.04$ & 3.69 \\
\hline Smith-Warner $(2003)^{(48)} /$ female+male/full cohort & 0.80 & $0.68,0.94$ & $7 \cdot 11$ \\
\hline Wright $(2008)^{(36)} /$ male/current smokers & 0.76 & $0.62,0.94$ & $5 \cdot 12$ \\
\hline Wright $(2008)^{(36)} /$ male/never smokers & 0.75 & $0.45,1.26$ & $1 \cdot 10$ \\
\hline Arts $(2001)^{(43)} /$ male/full cohort & 0.67 & $0 \cdot 38,1 \cdot 17$ & 0.93 \\
\hline Feskanich $(2000)^{(45)} /$ female/full cohort & 0.63 & $0.43,0.91$ & 1.97 \\
\hline Knekt $(2002)^{(42)} /$ male/full cohort & $0 \cdot 40$ & $0.22,0.74$ & 0.81 \\
\hline Overall (random-effects model) & 0.89 & $0.84,0.94$ & $100 \cdot 00$ \\
\hline
\end{tabular}

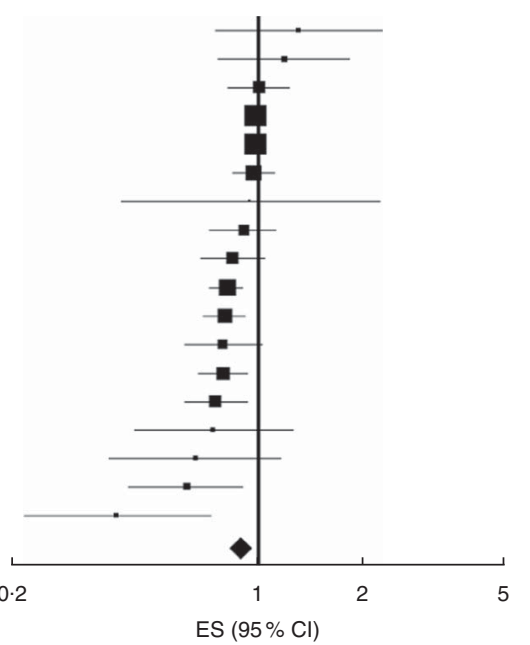

Fig. 2 Forest plots of case-control (a) and cohort (b) studies on apple consumption (highest $v$. lowest category) and lung cancer risk. Squares indicate the study-specific effect size (ES) derived from comparison between the highest and the lowest apple intake (size of square reflects the study's statistical weight, i.e. inverse of variance); horizontal lines indicate $95 \%$ confidence interval; diamond indicates the summary effect size estimate with its corresponding $95 \%$ confidence interval

retrospective studies should be interpreted with caution. In any event, when data from case-control studies were combined with those from cohort studies the metaanalysis showed a significant reduction in risk of lung (12\%), colorectal (28\%), oesophageal (34\%), digestive tract ( $41 \%)$ and breast (11\%) cancer.

Regardless of the absolute value of the effect size, the inverse association between apple intake and cancer risk is biologically plausible. Apples are a rich source of many different bioactive phenolic compounds (hydroxybenzoic and hydroxycinnamic acids, flavonols, dihydrochalcones, anthocyanids, monomeric and oligomeric flavanols) which may prevent cancer by several mechanisms ${ }^{(7)}$. First, phenols have a potent antioxidant activity which may protect DNA from oxidative damage. It has been estimated that a $100 \mathrm{~g}$ portion of apples has an antioxidant activity equal to $1.500 \mathrm{mg}$ of vitamin C. In addition, in vitro studies have demonstrated that apple phenols are able to inhibit tumour cell proliferation, induce cell cycle arrest and apoptosis, suppress angiogenesis and metastasis, modulate carcinogen metabolism and signal transduction pathways, and enhance the immune system. Accordingly, cancer chemopreventive properties of apple in vivo have also been demonstrated on several animal models for chemically or genetically induced tumours of the skin, breast and colon, as well as in xenograft models for solid tumours. These data demonstrate that apple constituents may have a systemic effect at the level of different organs, in addition to the more reasonable effect on the gastrointestinal tract.

In this regard, our results on the preventive effect of apples on lung cancer are very consistent in both case-control and cohort studies, even if in this last case a significant publication bias was found by the Egger test. Obviously, smoking status greatly influences lung cancer risk; therefore it would be important to stratify the analysis according to this variable. Unfortunately, only one case-control $^{(17)}$ and two cohort ${ }^{(36,38)}$ studies reported the risk of lung cancer in association with apple intake for smokers and non-smokers separately. Using these few data, we found a statistically significant effect of apple intake on lung cancer risk in current smokers but not in never smokers. Therefore, further studies are necessary to clarify the potential preventive effects of apples on lung cancer in smokers and never smokers. In contrast to lung cancer, the risk of colorectal and breast cancer resulted to 
Table 3 Results of stratified analysis of the risk estimates for the highest compared with the lowest apple intake on the basis of study type and cancer site ${ }^{\star} \dagger$

\begin{tabular}{|c|c|c|c|c|c|c|c|c|c|}
\hline \multirow[b]{2}{*}{ Cancer site } & \multicolumn{3}{|c|}{ Combined risk estimate } & \multicolumn{3}{|c|}{ Test of heterogeneity } & \multicolumn{2}{|c|}{ Publication bias } & \multirow[b]{2}{*}{ References } \\
\hline & Value & $95 \% \mathrm{Cl}$ & $P$ & $Q$ & $I^{2}(\%)$ & $P$ & $P$ (Egger's test) & $P($ Begg test $)$ & \\
\hline \multicolumn{10}{|l|}{ Lung } \\
\hline Case-control $(n 6) \ddagger$ & 0.75 & $0.63,0.88$ & 0.001 & 2.92 & 0.00 & 0.713 & 0.222 & 0.091 & $11,17,25$ \\
\hline Cohort $(n 18)$ & 0.89 & $0.84,0.94$ & $<0.001$ & 52.96 & 67.90 & $<0.001$ & 0.003 & 0.970 & $32,36,38,42,43,45,48$ \\
\hline Pooled§ ( $n$ 24) & 0.88 & $0.83,0.92$ & $<0.001$ & $65 \cdot 76$ & 65.02 & $<0.001$ & $<0.001$ & 1.000 & \\
\hline Current smokers $(n 6)$ & 0.88 & $0.77,1.00$ & 0.042 & 13.02 & 61.60 & 0.023 & 0.035 & 0.573 & $17,32,36$ \\
\hline Never-smokers $(n 5)$ & 0.88 & $0.67,1.15$ & 0.346 & 4.61 & 13.70 & 0.330 & 0.724 & 0.624 & $17,36,38$ \\
\hline Former smokers $(n 3)$ & 0.88 & $0.75,1.04$ & 0.137 & $3 \cdot 10$ & 35.54 & 0.212 & 0.041 & 0.117 & 36,38 \\
\hline Smokers + non-smokers $(n 10)$ & 0.82 & $0.72,0.92$ & 0.001 & 37.75 & $76 \cdot 16$ & $<0.001$ & 0.001 & 0.421 & $11,25,32,36,38,42,43,45,48$ \\
\hline Female $(n 7)$ & 0.92 & $0.82,1.03$ & 0.146 & 8.10 & 25.95 & 0.231 & 0.550 & 0.881 & $17,36,45$ \\
\hline Male $(n 8)$ & 0.79 & $0.73,0.85$ & $<0.001$ & $7 \cdot 18$ & 2.57 & 0.410 & 0.015 & 0.006 & $17,36,42,43$ \\
\hline Female + male $(n 9)$ & 0.95 & $0.90,1.00$ & 0.053 & 17.88 & 55.25 & 0.022 & 0.033 & 0.835 & $11,25,32,38,48$ \\
\hline \multicolumn{10}{|l|}{ Colorectal } \\
\hline Case-control ( $n 5)$ & 0.66 & $0.54,0.81$ & $<0.001$ & 8.89 & 54.99 & 0.064 & 0.594 & 0.327 & $14,16,20,22,28$ \\
\hline Cohort $(n 3)$ & 0.93 & $0.79,1.10$ & 0.400 & $2 \cdot 30$ & 13.06 & 0.317 & 0.169 & 0.117 & 39,49 \\
\hline Pooled§ $(n 8)$ & 0.72 & $0.59,0.88$ & 0.001 & 30.59 & 77.11 & $<0.001$ & 0.252 & 0.621 & \\
\hline \multicolumn{10}{|l|}{ Oral cavity } \\
\hline Case-control (n 3$)$ & 0.25 & $0.08,0.77$ & 0.015 & 70.29 & 97.15 & $<0.001$ & 0.273 & 0.117 & $21,22,24$ \\
\hline \multicolumn{10}{|l|}{ Oesophagus } \\
\hline Case-control (n 2) & 0.75 & $0.5,1.05$ & 0.091 & 0.99 & 0.00 & 0.319 & _- & _ & 15,22 \\
\hline Cohort $(n 2)$ & 0.60 & $0.21,1.71$ & 0.340 & 8.28 & 87.93 & 0.004 & - & - & 37 \\
\hline Pooled§ $(n 4)$ & 0.66 & $0.41,1.05$ & 0.078 & 9.30 & 67.74 & 0.026 & 0.277 & 0.497 & \\
\hline \multicolumn{10}{|l|}{ Overall digestive tractll } \\
\hline Case-control (n 10) & 0.50 & $0.36,0.69$ & $<0.001$ & 91.30 & $90 \cdot 14$ & $<0.001$ & 0.176 & 0.128 & $14-16,20-22,24,28$ \\
\hline Cohort $(n 6)$ & 0.79 & $0.61,1.01$ & 0.063 & $12 \cdot 84$ & 61.05 & 0.025 & 0.069 & 0.005 & $37,39,46,49$ \\
\hline Polled $\S(n 16)$ & 0.59 & $0.47,0.74$ & $<0.001$ & 136.02 & 88.97 & $<0.001$ & 0.048 & 0.072 & \\
\hline \multicolumn{10}{|l|}{ Breast } \\
\hline Case-control ( $n$ 5) & 0.79 & $0.73,0.87$ & $<0.001$ & 4.04 & 0.88 & 0.401 & 0.585 & 0.327 & $12,18,22,23,26$ \\
\hline Cohort $(n 3)$ & 0.97 & $0.94,1.01$ & 0.192 & 0.92 & 0.00 & 0.631 & 0.135 & 0.117 & $30,40,47$ \\
\hline Pooled§ (n 8) & 0.89 & $0.79,1.00$ & 0.047 & $22 \cdot 37$ & 68.71 & 0.002 & 0.228 & 0.805 & \\
\hline \multicolumn{10}{|l|}{ Prostate } \\
\hline Case-control (n 2) & 0.93 & $0.79,1.09$ & 0.369 & 0.19 & 0.00 & 0.664 & _- & _- & 10,22 \\
\hline
\end{tabular}

${ }^{*}$ The analysis was performed when two or more studies were available

The risk estimates were calculated using the random-effect model.

fNumber of studies used to calculate the risk is indicated in parentheses.

Analysis was performed on case-control and cohort studies combined together.

IIColorectal, oral cavity, oesophageal and stomach cancers. 
(a)

\begin{tabular}{|c|c|c|c|}
\hline & ES & $95 \% \mathrm{Cl}$ & Weight (\%) \\
\hline Theodoratou (2007) & 0.96 & $0.62,1.50$ & $14 \cdot 18$ \\
\hline Annema $(2011)^{(14)}$ & 0.74 & $0.56,0.99$ & $22 \cdot 69$ \\
\hline Gallus $(2005)^{(22)}$ & $0 \cdot 70$ & $0.62,0.79$ & 34.93 \\
\hline Jedrychowski $(2010)^{(16)}$ & 0.53 & $0.35,0.79$ & $15 \cdot 70$ \\
\hline Deneo-Pellegrini $(1996)^{(28)}$ & $0 \cdot 40$ & $0.25,0.66$ & $12 \cdot 51$ \\
\hline ndom-effects model) & 0.66 & $0.54,0.81$ & 100.00 \\
\hline
\end{tabular}

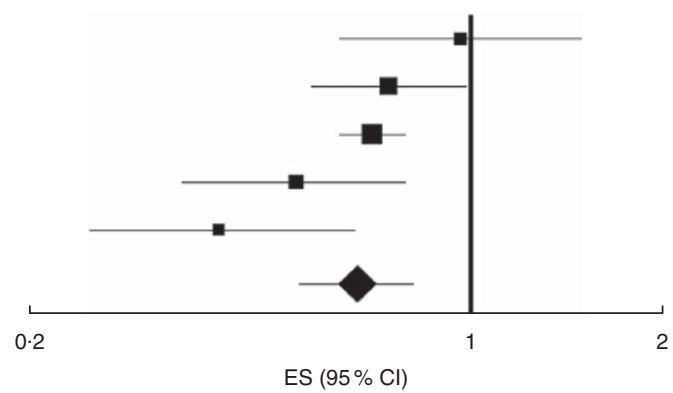

(b)

\begin{tabular}{rccc} 
& ES & $95 \% \mathrm{Cl}$ & Weight (\%) \\
\cline { 2 - 4 } Koushik $(2007)^{(49)} /$ male+female & 0.98 & $0.88,1 \cdot 10$ & $81 \cdot 18$ \\
Lin $(2006)^{(39)} /$ male & 0.82 & $0.51,1 \cdot 30$ & 11.59 \\
Lin $(2006)^{(39)} /$ female & 0.64 & $0.35,1 \cdot 17$ & $7 \cdot 23$ \\
Overall (random-effects model) & 0.93 & $0.79,1 \cdot 10$ & $100 \cdot 00$
\end{tabular}

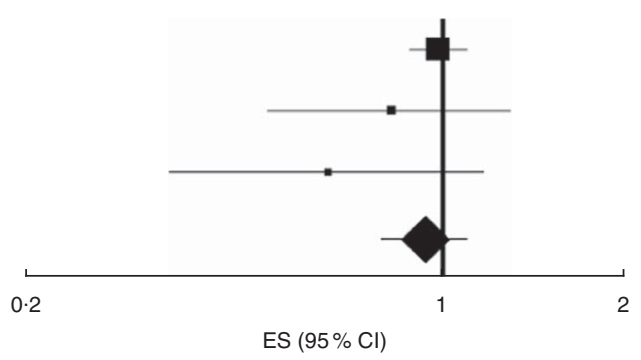

Fig. 3 Forest plots of case-control (a) and cohort (b) studies on apple consumption (highest $v$. lowest category) and colorectal cancer risk. Squares indicate the study-specific effect size (ES) derived from comparison between the highest and the lowest apple intake (size of square reflects the study's statistical weight, i.e. inverse of variance); horizontal lines indicate $95 \%$ confidence interval; diamond indicates summary effect size estimate with its corresponding $95 \%$ confidence interval

(a)

\begin{tabular}{|c|c|c|c|}
\hline & ES & $95 \% \mathrm{Cl}$ & Weight (\%) \\
\hline Malin (2003) $)^{(23)}$ & 0.86 & $0 \cdot 66,1 \cdot 11$ & 11.57 \\
\hline Bao $(2012)^{(12)}$ & 0.84 & $0.71,0.98$ & $29 \cdot 78$ \\
\hline Torres-Sánchez (2000) ${ }^{(26)}$ & 0.83 & $0.54,1.28$ & $4 \cdot 22$ \\
\hline Gallus $(2005)^{(22)}$ & 0.76 & $0.67,0.85$ & $53 \cdot 86$ \\
\hline Di Pietro $(2007)^{(18)}$ & 0.30 & $0.09,0.94$ & 0.57 \\
\hline od & 0.79 & $0.73,0.87$ & $100 \cdot 00$ \\
\hline
\end{tabular}

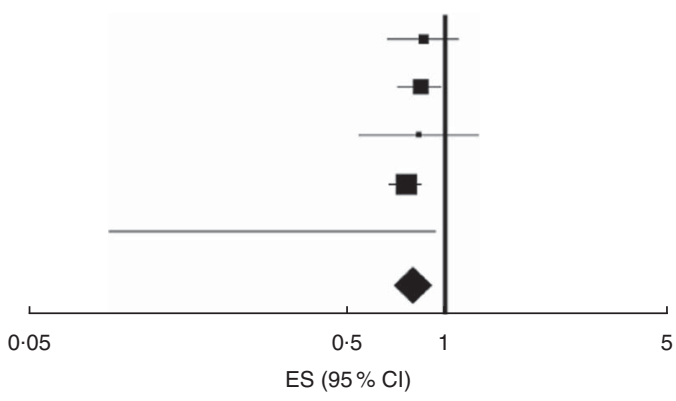

(b)

\begin{tabular}{|c|c|c|c|}
\hline & ES & $95 \% \mathrm{Cl}$ & Weight (\%) \\
\hline Adebamovo $(2005)^{(40)}$ & $1 \cdot 16$ & $0.77,1.76$ & 0.95 \\
\hline Boggs $(2010)^{(30)}$ & 1.02 & $0.83,1.25$ & 3.87 \\
\hline Smith-Warner $(2001)^{(47)}$ & 0.97 & $0.93,1.01$ & $95 \cdot 19$ \\
\hline (random-effects model) & 0.97 & $0.94,1.01$ & $100 \cdot 00$ \\
\hline
\end{tabular}

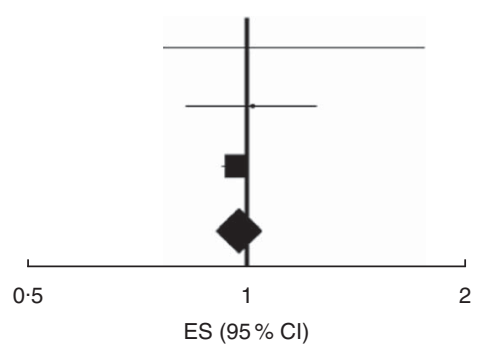

Fig. 4 Forest plots of case-control (a) and cohort (b) studies on apple consumption (highest $v$. lowest category) and breast cancer risk. Squares indicate the study-specific effect size (ES) derived from comparison between the highest and the lowest apple intake (size of square reflects the study's statistical weight, i.e. inverse of variance); horizontal lines indicate $95 \%$ confidence interval; diamond indicates summary effect size estimate with its corresponding $95 \%$ confidence interval

be significantly reduced in case-control studies $(34 \%$ and $21 \%$, respectively) while no significant effect was found in cohort studies. These discrepancies may be in part due to the low number of cohort studies, two for colonrectum $^{(39,49)}$ and three for breast ${ }^{(30,40,47)}$. It should be considered, however, that two pooled analyses were 
included in our meta-analysis, one on colorectal cancer ${ }^{(49)}$ and the other on breast cancer ${ }^{(47)}$, which considered eleven and seven cohort studies, respectively. Furthermore, a recent pooled analysis of twenty cohort studies on breast cancer (not included in our meta-analysis) showed a small but significant effect ( $R R=0 \cdot 92 ; 95 \%$ CI 0.85, 0.99) of apples, together with pears and applesauce, on oestrogen receptor-negative breast cancer ${ }^{(53)}$. Therefore, as a result of the complexity and variety of the carcinogenic process, it is possible that the stratification of cases according to molecular targets may highlight associations that until now have not been considered. Further studies are needed to investigate these aspects.

There were some limitations in our meta-analysis. Heterogeneity was evident and, in some cases, particularly high. One reason for this could be the wide range of values for the cut-off points for the lowest and highest categories of apple intake. In addition, the number of studies included in the meta-analysis for each cancer type was not large enough to stratify the analysis according to geographic region, sex and adjustment for confounding factors to try to determine the source of heterogeneity. In the case of lung cancer risk, publication bias was also detected in cohort studies. Stratification according to smoking and sex did not clearly reduce both heterogeneity and publication bias in studies on lung cancer. However, the exclusion of one study ${ }^{(32)}$ did not significantly modify the lung cancer risk estimate but eliminated both heterogeneity and publication bias. Although when multiple estimates were available, we abstracted those that adjusted for the most confounding factors, many of the studies included in the analysis varied in the number of potential diet confounding variables (i.e. meat, dairy products, fibre) for which they had not been adjusted. Furthermore, most of the studies were not designed solely to evaluate the association between apple consumption and cancer risk and there were wide variations in dietary assessments of the frequency/quantity of apple intake. For these reasons, in addition to the low number of data available for each cancer site, it was not possible to calculate the dose-response relationship between apple intake and cancer risk in different anatomical organs.

\section{Conclusion}

In summary, the current meta-analysis provides convincing evidence supporting the hypothesis of the protective ability of apples in the aetiology of cancer. However, some confounding effects may be present and related to the consumption of other fruit which have not been considered as adjusting factors. Apple consumption was associated with a reduced risk of cancer in the lung, colon-rectum, oral cavity, digestive tract and breast. Further studies will be needed to clarify the effect of apples on cancer risk in other anatomical sites.

\section{Acknowledgements}

Financial support: All work was completed at the University of Perugia, Italy. The authors thank their home institution for financial support. Conflict of interest: The authors declare that they have no conflict of interest. Authorship: Study concept and design: R.F., L.M. and P.R. Acquisition of data: R.F. and M.L. Analysis and interpretation of data: R.F., L.M. and P.R. All authors contributed substantively to this manuscript, were involved with critical revisions to the manuscript and provided approval for its publication. Ethics of human subject participation: Not applicable.

\section{Supplementary material}

To view supplementary material for this article, please visit http://dx.doi.org/10.1017/S136898001600032X

\section{References}

1. Ferlay J, Soerjomataram I, Ervik M et al. (2013) GLOBOCAN 2012 v1.0, Cancer Incidence and Mortality Worldwide: IARC CancerBase No. 11. Lyon: International Agency for Research on Cancer; available at http:// globocan.iarc.fr

2. Vineis P \& Wild CP (2014) Global cancer patterns: causes and prevention. Lancet 383, 549-557.

3. Willett WC (1995) Diet, nutrition, and avoidable cancer. Environ Health Perspect 103, 165-170.

4. Liu RH (2013) Health-promoting components of fruits and vegetables in the diet. Adv Nutr 4, issue 3, 384S-392S.

5. Norat T, Aune D, Chan D et al. (2014) Fruits and vegetables: updating the epidemiologic evidence for the WCRF/AICR lifestyle recommendations for cancer prevention. Cancer Treat Res 159, 35-50.

6. World Cancer Research Fund/American Institute for Cancer Research (2007) Food, Nutrition and the Prevention of Cancer: A Global Perspective. Washington, DC: American Institute for Cancer Research.

7. Hyson DA (2011) A comprehensive review of apples and apple components and their relationship to human health. Adv Nutr 2, 408-420.

8. Stroup DF, Berlin JA, Morton SC et al. (2000) Meta-analysis of observational studies in epidemiology: a proposal for reporting. Meta-analysis Of Observational Studies in Epidemiology (MOOSE) group. JAMA 283, 2008-2012.

9. Wells GA, Shea B, O'Connell D et al. (2014) The NewcastleOttawa Scale (NOS) for assessing the quality of nonrandomised studies in meta-analyses. http://www.ohri. $\mathrm{ca} /$ programs/clinical_epidemiology/oxford.asp (accessed July 2015).

10. Askari F, Parizi MK, Jessri M et al. (2014) Fruit and vegetable intake in relation to prostate cancer in Iranian men: a case-control study. Asian Pac J Cancer Prev 15, 5223-5227.

11. Tarrazo-Antelo AM, Ruano-Ravina A, Abal Arca J et al. (2014) Fruit and vegetable consumption and lung cancer risk: a case-control study in Galicia, Spain. Nutr Cancer $\mathbf{6 6}$, 1030-1037.

12. Bao PP, Shu XO, Zheng Y et al. (2012) Fruit, vegetable, and animal food intake and breast cancer risk by hormone receptor status. Nutr Cancer 64, 806-819.

13. Rossi M, Lugo A, Lagiou P et al. (2012) Proanthocyanidins and other flavonoids in relation to pancreatic cancer: a case-control study in Italy. Ann Oncol 23, 1488-1493. 
14. Annema N, Heyworth JS, McNaughton SA et al. (2011) Fruit and vegetable consumption and the risk of proximal colon, distal colon, and rectal cancers in a case-control study in Western Australia. J Am Diet Assoc 111, 1479-1490.

15. Hajizadeh B, Jessri M, Moasheri SM et al. (2011) Fruits and vegetables consumption and esophageal squamous cell carcinoma: a case-control study. Nutr Cancer 63, 707-713.

16. Jedrychowski W, Maugeri U, Popiela T et al. (2010) Casecontrol study on beneficial effect of regular consumption of apples on colorectal cancer risk in a population with relatively low intake of fruits and vegetables. Eur J Cancer Prev 19, 42-47.

17. Kubik A, Zatloukal P, Tomasek L et al. (2008) A casecontrol study of lifestyle and lung cancer associations by histological types. Neoplasma 55, 192-199.

18. Di Pietro PF, Medeiros NI, Vieira FG et al. (2007) Breast cancer in southern Brazil: association with past dietary intake. Nutr Hosp 22, 565-572.

19. Sacerdote C, Matullo G, Polidoro S et al. (2007) Intake of fruits and vegetables and polymorphisms in DNA repair genes in bladder cancer. Mutagenesis 22, 281-285.

20. Theodoratou E, Kyle J, Cetnarskyj R et al. (2007) Dietary flavonoids and the risk of colorectal cancer. Cancer Epidemiol Biomarkers Prev 16, 684-693.

21. Kreimer AR, Randi G, Herrero R et al. (2006) Diet and body mass, and oral and oropharyngeal squamous cell carcinomas: analysis from the IARC multinational casecontrol study. Int J Cancer 118, 2293-2297.

22. Gallus S, Talamini R, Giacosa A et al. (2005) Does an apple a day keep the oncologist away? Ann Oncol 16, 1841-1844.

23. Malin AS, Qi D, Shu XO et al. (2003) Intake of fruits, vegetables and selected micronutrients in relation to the risk of breast cancer. Int J Cancer 105, 413-418.

24. Rajkumar T, Sridhar H, Balaram P et al. (2003) Oral cancer in Southern India: the influence of body size, diet, infections and sexual practices. Eur J Cancer Prev 12, 135-143.

25. Le Marchand L, Murphy SP, Hankin JH et al. (2000) Intake of flavonoids and lung cancer. J Natl Cancer Inst 92, 154-160.

26. Torres-Sánchez L, López-Carrillo L, López-Cervantes M et al. (2000) Food sources of phytoestrogens and breast cancer risk in Mexican women. Nutr Cancer 37, 134-139.

27. Lindblad P, Wolk A, Bergström R et al. (1997) Diet and risk of renal cell cancer: a population-based case-control study. Cancer Epidemiol Biomarkers Prev 6, 215-223.

28. Deneo-Pellegrini H, De Stefani E \& Ronco A (1996) Vegetables, fruits, and risk of colorectal cancer: a casecontrol study from Uruguay. Nutr Cancer 25, 297-304.

29. Giles GG, McNeil JJ, Donnan G et al. (1994) Dietary factors and the risk of glioma in adults: results of a case-control study in Melbourne, Australia. Int J Cancer 59, 357-362.

30. Boggs DA, Palmer JR, Wise LA et al. (2010) Fruit and vegetable intake in relation to risk of breast cancer in the Black Women's Health Study. Am J Epidemiol 172, 1268-1279.

31. Kabat GC, Park Y, Hollenbeck AR et al. (2010) Intake of fruits and vegetables, and risk of endometrial cancer in the NIH-AARP Diet and Health Study. Cancer Epidemiol 34, 568-573.

32. Büchner FL, Bueno-de-Mesquita HB, Linseisen $\mathrm{J}$ et al. (2010) Fruits and vegetables consumption and the risk of histological subtypes of lung cancer in the European Prospective Investigation into Cancer and Nutrition (EPIC). Cancer Causes Control 21, 357-371.

33. Büchner FL, Bueno-de-Mesquita HB, Ros MM et al. (2009) Consumption of vegetables and fruit and the risk of bladder cancer in the European Prospective Investigation into Cancer and Nutrition. Int J Cancer 125, 2643-2651.

34. Wang L, Lee IM, Zhang SM et al. (2009) Dietary intake of selected flavonols, flavones, and flavonoid-rich foods and risk of cancer in middle-aged and older women. Am J Clin Nutr 89, 905-912.

35. Freedman ND, Park Y, Subar AF et al. (2008) Fruit and vegetable intake and head and neck cancer risk in a large United States prospective cohort study. Int J Cancer 122, 2330-2336.

36. Wright ME, Park Y, Subar AF et al. (2008) Intakes of fruit, vegetables, and specific botanical groups in relation to lung cancer risk in the NIH-AARP Diet and Health Study. Am J Epidemiol 168, 1024-1034.

37. Freedman ND, Park Y, Subar AF et al. (2007) Fruit and vegetable intake and esophageal cancer in a large prospective cohort study. Int J Cancer 121, 2753-2760.

38. Linseisen J, Rohrmann S, Miller AB et al. (2007) Fruit and vegetable consumption and lung cancer risk: updated information from the European Prospective Investigation into Cancer and Nutrition (EPIC). Int $J$ Cancer 121, 1103-1114.

39. Lin J, Zhang SM, Wu K et al. (2006) Flavonoid intake and colorectal cancer risk in men and women. Am J Epidemiol 164, 644-651

40. Adebamowo CA, Cho E, Sampson L et al. (2005) Dietary flavonols and flavonol-rich foods intake and the risk of breast cancer. Int J Cancer 114, 628-633.

41. Rashidkhani B, Lindblad P \& Wolk A (2005) Fruits, vegetables and risk of renal cell carcinoma: a prospective study of Swedish women. Int J Cancer 113, 451-455.

42. Knekt P, Kumpulainen J, Järvinen R et al. (2002) Flavonoid intake and risk of chronic diseases. Am J Clin Nutr 76, 560-568.

43. Arts IC, Hollman PC, Bueno De Mesquita HB et al. (2001) Dietary catechins and epithelial cancer incidence: the Zutphen elderly study. Int J Cancer 92, 298-302.

44. Zeegers MP, Goldbohm RA \& van den Brandt PA (2001) Consumption of vegetables and fruits and urothelial cancer incidence: a prospective study. Cancer Epidemiol Biomarkers Prev 10, 1121-1128.

45. Feskanich D, Ziegler RG, Michaud DS et al. (2000) Prospective study of fruit and vegetable consumption and risk of lung cancer among men and women. J Natl Cancer Inst 92, 1812-1823.

46. Botterweck AA, van den Brandt PA \& Goldbohm RA (1998) A prospective cohort study on vegetable and fruit consumption and stomach cancer risk in The Netherlands. Am J Epidemiol 148, 842-853.

47. Smith-Warner SA, Spiegelman D, Yaun SS et al. (2001) Intake of fruits and vegetables and risk of breast cancer: a pooled analysis of cohort studies. JAMA 285, 769-776.

48. Smith-Warner SA, Spiegelman D, Yaun SS et al. (2003) Fruits, vegetables and lung cancer: a pooled analysis of cohort studies. Int J Cancer 107, 1001-1011.

49. Koushik A, Hunter DJ, Spiegelman D et al. (2007) Fruits, vegetables, and colon cancer risk in a pooled analysis of 14 cohort studies. J Natl Cancer Inst 99, 1471-1483.

50. Koushik A, Spiegelman D, Albanes D et al. (2012) Intake of fruits and vegetables and risk of pancreatic cancer in a pooled analysis of 14 cohort studies. Am J Epidemiol 176, 373-386.

51. Riboli E \& Norat T (2003) Epidemiologic evidence of the protective effect of fruit and vegetables on cancer risk. $A m J$ Clin Nutr 78, 3 Suppl., 559S-569S.

52. Kaaks R \& Riboli E (2005) Epidemiologic studies of nutrition and cancer: let us not throw out the baby with the bath water. Int J Cancer 116, 662-664.

53. Jung S, Spiegelman D, Baglietto L et al. (2013) Fruit and vegetable intake and risk of breast cancer by hormone receptor status. J Natl Cancer Inst 105, 219-236. 